\title{
Venoarterial extracorporeal membrane oxygenation with or without simultaneous intra-aortic balloon pump support as a direct bridge to heart transplantation: results from a nationwide Spanish registry
}

Gonzalo Barge-Caballero ${ }^{\mathrm{a}, \mathrm{b}}$, María A Castel-Lavilla ${ }^{\mathrm{c}}$, Luis Almenar-Bonet ${ }^{\mathrm{d}}$, Iris P Garrido-Bravo $^{\mathrm{b}, \mathrm{e}}$, Juan F Delgadob,f, Diego Rangel-Sousa ${ }^{\mathrm{g}}$, José González-Costello ${ }^{\mathrm{h}}$, Javier Segovia-Cubero ${ }^{\mathrm{b}, \mathrm{i}}$, Marta Farrero-Torres ${ }^{\mathrm{c}}$, José Luis Lambert-Rodríguez,k, María G Crespo-Leiro a,b, Daniela Hervás-Sotomayor ${ }^{\mathrm{l}}$, Ana Portolés-Ocampom, Manuel Martínez-Sellés $^{\mathrm{b}, \mathrm{n}}$, Luis De la Fuente-Galán ${ }^{\mathrm{b}, o}$, Gregorio Rábago-Juan-Aracil ${ }^{\mathrm{p}}$, Francisco González-Vílchez ${ }^{\mathrm{q}}$, Sonia Mirabet-Pérez ${ }^{\mathrm{r}}$, Javier Muñiz ${ }^{\mathrm{b}, \mathrm{s}}$, Eduardo BargeCaballero $^{\mathrm{a}, \mathrm{b}}$

a Complejo Hospitalario Universitario A Coruña (CHUAC), Instituto de Investigación Biomédica de A Coruña (INIBIC), A Coruña, Spain

b Centro de Investigación Biomédica en Red de Enfermedades Cardiovasculares (CIBERCV), Instituto de Salud Carlos III, Madrid, Spain

Hospital Clinic i Provincial, Barcelona, Spain

d Hospital Universitario y Politécnico La Fe, Valencia, Spain

Hospital Universitario Virgen de la Arrixaca, Murcia, Spain

Hospital Universitario Doce de Octubre, Instituto de Investigación i+12, Facultad de Medicina, Universidad Complutense de Madrid, Madrid, Spain

$g \quad$ Hospital Universitario Virgen del Rocío, Sevilla, Spain

hospital Universitario de Bellvitge, Hospitalet de Llobregat, Spain

Hospital Universitario Puerta de Hierro, Majadahonda, Spain

Hospital Universitario Central de Asturias, Oviedo, Spain

Universidad Católica San Antonio, Murcia, Spain

Hospital Universitario Reina Sofía, Córdoba, Spain

m Hospital Universitario Miguel Servet, Zaragoza, Spain

n Hospital General Universitario Gregorio Marañón, Universidad Complutense, Universidad Europea, Madrid, Spain

- Hospital Clínico Universitario, Valladolid, Spain

p Clínica Universidad de Navarra, Pamplona, Spain

q Hospital Universitario Marqués de Valdecilla, Santander, Spain

Hospital Santa Creu i Sant Pau, Barcelona, Spain

$s$ Instituto Universitario de Ciencias de la Salud, Universidad de A Coruña (UDC), INIBIC, A Coruña, Spain 


\section{Abstract}

\section{Objectives}

To investigate the potential clinical benefit of an intra-aortic balloon pump (IABP) in patients supported with venoarterial extracorporeal membrane oxygenation (VA-ECMO) as a bridge to heart transplantation (HT)

\section{Methods}

We studied 169 patients who were listed for urgent HT under VA-ECMO support at 16 Spanish institutions from 2010 to 2015. The clinical outcomes of patients under simultaneous IABP support $(n=73)$ were compared to a control group of patients without IABP support $(n=96)$.

\section{Results}

There were no statistically significant differences between the IABP and control groups with regard to the cumulative rates of transplantation $(71.2 \%$ vs $81.2 \%, \mathrm{P}=0.17)$, death during VA-ECMO support (20.6\% vs $14.6 \%$, $\mathrm{P}=0.31)$, transition to a different mechanical circulatory support device $(5.5 \% \mathrm{vs} 5.2 \%, \mathrm{P}=0.94)$ or weaning from VA-ECMO support due to recovery $(2.7 \%$ vs $0 \%, \mathrm{P}=0.10)$. There was a higher incidence of bleeding events in the IABP group ( $45.2 \%$ vs $25 \%, \mathrm{P}=0.006$; adjusted odds ratio $2.18,95 \%$ confidence interval $1.02-4.67)$. In-hospital postoperative mortality after HT was $34.6 \%$ in the IABP group and $32.5 \%$ in the control group $(\mathrm{P}=0.80)$. One-year survival after listing for urgent HT was 53.3\% in the IABP group and 52.2\% in the control group $(\log \operatorname{rank} \mathrm{P}=0.75)$. Multivariate adjustment for potential confounders did not change this result (adjusted hazard ratio 0.94, 95\% confidence interval $0.56-1.58$ ).

\section{Conclusions}

In our study, simultaneous IABP therapy in transplant candidates under VA-ECMO support did not significantly reduce morbidity or mortality.

Keywords

Heart transplantation, Extracorporeal membrane oxygenation, Intra-aortic balloon pump

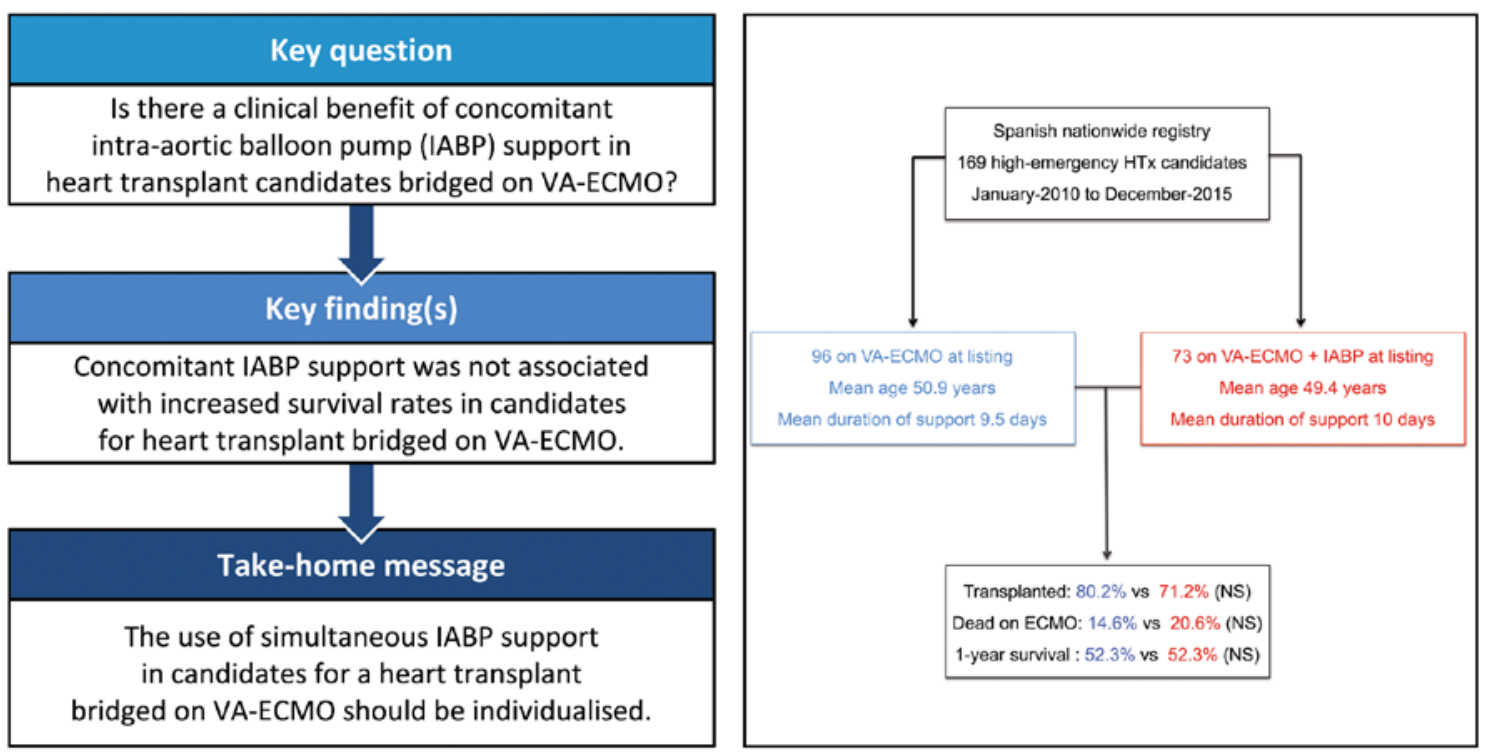




\section{Introduction}

Heart transplantation (HT) is the therapy of choice for patients with refractory heart failure [1]. Given the scarcity of donors, an increasing number of candidates undergo HT under a high-urgency indication [2], which frequently requires preoperative mechanical circulatory support [3].

Given the peculiarities of the Spanish organ-sharing network, venoarterial extracorporeal membrane oxygenation (VA-ECMO) is commonly used as a direct bridge to high-urgency HT for critically ill candidates [2, 3]. The main advantage of this device is its easy implantation, which enables the immediate initiation of advanced circulatory and respiratory support through percutaneous access. Therefore, VAECMO is especially useful for the initial management of patients in cardiogenic shock or cardiac arrest.

Left ventricular distension and pulmonary congestion due to increased afterload are frequent complications in patients supported with VA-ECMO [4]. It has been suggested that the ancillary use of an intra-aortic balloon pump (IABP) in these individuals might lead to improved unloading of the left ventricle, which could help prevent hydrostatic pulmonary oedema [5]. However, previous studies have reported controversial results regarding the impact of this strategy in terms of survival [6-9].

The aim of our investigation was to determine the efficacy and safety of the ancillary use of an IABP in patients supported with VA-ECMO, with a primary intention of the bridge to transplantation. Previous multicentre studies reporting the results of VA-ECMO in this situation did not address this specific question $[10,11]$.

\section{Methods}

\section{Study description}

The ASIS-TC study [12], was a retrospective multicentre registry that included all consecutive patients who were treated with temporary mechanical circulatory support devices, and subsequently listed for first, single-organ, high-urgency HT in Spain between 2010 and 2015. All of the 16 adult HT centres of Spain were involved in the registry. The study protocol was approved by the Committee for Ethics in Clinical Research of the Autonomous Community of Galicia, Spain, and ratified by the institutional review boards of all participating institutions.

In this article, we describe the clinical outcomes of patients in the ASIS-TC registry who received simultaneous support with VA-ECMO and IABP, as compared to patients who were supported solely with VA-ECMO.

\section{Outcomes}

The primary outcome of this study was 1-year survival after listing for high-urgency HT. Secondary outcomes included the cumulative incidence of death from any cause, HT, weaning from VA-ECMO due to clinical improvement ('recovery') and transition to a different mechanical device during the in-hospital period following listing, and the cumulative incidence of adverse clinical events during VA-ECMO support. Device dysfunction, bleeding events, stroke and device dysfunction were considered major adverse clinical events during VA-ECMO support. Complete follow-up was available for all patients.

In patients who underwent high-urgency HT, while on VA-ECMO support, we also analysed the cumulative incidence of postoperative death and postoperative adverse clinical events until hospital discharge. For this analysis, patients were considered to have been treated simultaneously with VAECMO and IABP if both devices were in place and functioning at the time of listing, even if the IABP was removed during the waiting period before transplantation.

Specific definitions of all study outcomes are detailed in the Supplementary Material. 


\section{Statistical analysis}

In this article, quantitative variables are expressed as mean (standard deviation, SD), and categorical variables are expressed as proportions. For intergroup comparisons, the Student's t-test and the $\chi^{2}$ test were used as required.

The cumulative probability of death and adverse clinical events was represented graphically by means of Kaplan-Meier curves, which were statistically compared using the log-rank test.

The multivariable Cox's regression analysis was performed to control the effect of confusion bias on the statistical association between IABP use and 1-year survival. In a first step, we calculated the univariate hazard ratios for the outcome of interest for all baseline clinical variables listed in Table 1 that showed a statistically significant or near-significant $(\mathrm{P}<0.010)$ different distribution between the IABP and control groups. Then, those variables that also showed a statistically significant univariate association with 1-year survival entered the final multivariable model, to calculate the adjusted hazard ratio for 1-year survival for IABP support versus control. Following a similar procedure, multivariable logistic regression was used to assess confusion bias regarding the risk of bleeding events.

Statistical analyses were performed with SPSS 20. Statistical significance was set at a P-value of $<0.05$.

\section{Results}

Patients

Between January 2010 and December 2015, 169 patients supported with VA-ECMO were listed for high-urgency HT in 16 Spanish institutions. Seventy-three (43\%) patients were simultaneously supported with an IABP at the time of listing (IABP group). In 68 of them, the IABP had been inserted before VAECMO implantation and was maintained afterwards.

The remaining 96 (57\%) patients were supported solely with VA-ECMO at the time of listing for high-urgency HT (control group). Among them, 15 (16\%) had been supported with IABP before VAECMO, but the first device was removed after insertion of the oxygenator.

Patients supported simultaneously with VA-ECMO and IABP presented with shock related to a myocardial infarction more frequently than patients in the control group. In addition, a higher proportion of patients in the IABP group were treated with mechanical ventilation and vasoactive drugs (Table 1).

The proportion of patients who received double antiplatelet therapy was higher in the IABP group than in the control group; however, the mean values of haemoglobin concentration and international normalized ratio were significantly higher in the latter. Only 2 patients in each group had an apical vent for left ventricular unloading. No other therapies for left ventricular unloading, such as left ventricular assist device implantation or atrial septostomy, were used at baseline in the studied patients

\section{Outcomes of venoarterial extracorporeal membrane oxygenation support}

Twenty-nine (17.2\%) patients died and 126 (76.3\%) were transplanted, while under VA-ECMO support. Nine (5.3\%) patients transitioned from VA-ECMO to a different mechanical device: 6 patients to Centrimag ${ }^{\circledR}, 2$ patients to BerlinHeart Excor ${ }^{\circledR}$ and 1 patient to Maquet Rotaflow ${ }^{\circledR}$. Only 2 (1.2\%) patients had the VA-ECMO explanted due to recovery.

The mean (SD) duration of VA-ECMO support was 10 (6.3) days in the IABP group and 9.5 (9.2) days in the control group $(\mathrm{P}=0.68)$. No statistically significant differences between the IABP and control groups were observed with regard to the cumulative incidence of death from any cause (20.6\% vs $14.6 \%$, $\mathrm{P}=0.31)$, transplantation $(71.2 \%$ vs $80.2 \%, \mathrm{P}=0.17)$, transition to another mechanical device $(5.5 \% \mathrm{vs}$ $5.2 \%, \mathrm{P}=0.91)$ or weaning from VA-ECMO due to recovery $(2.7 \% \mathrm{vs} 0 \%, \mathrm{P}=0.19)$ during this support period (Fig. 1). 
Table 1: Baseline clinical characteristics of patients in both study groups

\begin{tabular}{|c|c|c|c|}
\hline & $\mathrm{VA}-\mathrm{ECMO}+\mathrm{IABP}(\mathrm{n}=73)$ & VA-ECMO alone $(\mathrm{n}=96)$ & P-value \\
\hline Age (years), mean (SD) & $49.4(12.7)$ & $50.9(13.3)$ & 0.46 \\
\hline Women, n (\%) & $18(24.7)$ & $23(24)$ & 0.92 \\
\hline Body mass index (kg/m2), mean (SD) & $25.3(5.1)$ & $26(4.2)$ & 0.39 \\
\hline \multicolumn{4}{|l|}{ Configuration of VA-ECMO } \\
\hline Arterial cannulation site & & & 0.049 \\
\hline Peripheral, femoral artery (\%) & $67(91.8)$ & $76(79.2)$ & \\
\hline Peripheral, subclavian artery (\%) & $3(4.1)$ & $15(15.6)$ & \\
\hline Central (\%) & $3(4.1)$ & $5(5.2)$ & \\
\hline Left ventricular apical venting, n (\%) & $2(2.7)$ & $2(2.1)$ & 0.78 \\
\hline Distal perfusion catheter, n (\%) & $45(61.6)$ & $74(77.1)$ & 0.029 \\
\hline \multicolumn{4}{|l|}{ Patient already listed before VA-ECMO } \\
\hline insertion, n (\%) & $12(16.4)$ & $35(36.5)$ & $<0.001$ \\
\hline Time from hospital admission to VA-ECMO & & & \\
\hline insertion (days), mean (SD) & $7.1(11.3)$ & $14.8(24.4)$ & 0.008 \\
\hline \multicolumn{4}{|l|}{ Time from VA-ECMO insertion to high-urgency } \\
\hline listing (days), mean (SD) & $2.8(2.7)$ & $2.5(5.7)$ & 0.71 \\
\hline Ischaemic heart disease, $\mathrm{n}(\%)$ & $45(61.6)$ & 45 (46.9) & 0.057 \\
\hline Shock secondary to acute myocardial infarction, n (\%) & $33(45.2)$ & $21(21.9)$ & 0.001 \\
\hline Postcardiotomy shock, n (\%) & $11(15.1)$ & $6(6.2)$ & 0.059 \\
\hline Cardiopulmonary arrest, n (\%) & $15(20.5)$ & $14(14.6)$ & 0.31 \\
\hline Previous sternotomy, n (\%) & $20(27.4)$ & $25(26)$ & 0.84 \\
\hline Diabetes mellitus, n (\%) & $17(23.3)$ & $26(27.1)$ & 0.58 \\
\hline Peripheral artery disease, n (\%) & $3(4.1)$ & $7(7.3)$ & 0.49 \\
\hline Chronic obstructive pulmonary disease, n (\%) & $3(4.1)$ & $4(4.2)$ & 0.99 \\
\hline Active infection requiring i.v. antibiotics, $\mathrm{n}(\%)$ & $7(9.6)$ & $4(4.2)$ & 0.16 \\
\hline INTERMACS profile 1 or $2(\%)$ & $69(94.5)$ & $86(89.6)$ & 0.29 \\
\hline Renal replacement therapy, n (\%) & $5(6.8)$ & $7(7.3)$ & 0.91 \\
\hline Invasive mechanical ventilation, $\mathrm{n}(\%)$ & $63(86.3)$ & $68(70.8)$ & 0.017 \\
\hline Vasoactive drug support, n (\%) & $71(97.3)$ & $81(85.3)$ & 0.009 \\
\hline Vasoactive inotropic score (units), mean (SD) & $58(84)$ & $29(38)$ & 0.012 \\
\hline \multicolumn{4}{|l|}{ Antiplatelet therapy, n (\%) } \\
\hline Aspirin & $19(26)$ & $32(33.3)$ & 0.31 \\
\hline Clopidogrel & $16(21.9)$ & $9(9.4)$ & 0.023 \\
\hline Other & $2(2.7)$ & 0 & 0.10 \\
\hline Type of antiplatelet therapy & 0.008 & & \\
\hline None & $50(68.5)$ & $62(64.6)$ & \\
\hline One drug & $9(12.3)$ & $27(28.1)$ & \\
\hline Two drugs & $14(19.2)$ & $7(7.3)$ & \\
\hline Haemoglobin (g/dl), mean (SD) & $10(1.8)$ & $10.8(2.1)$ & 0.015 \\
\hline Platelets $(103 \times \mu \mathrm{l})$, mean (SD) & $185(84)$ & $186(80)$ & 0.91 \\
\hline INR (UI), mean (SD) & $1.38(0.94)$ & $1.70(0.96)$ & 0.003 \\
\hline Creatinine (mg/dl), mean (SD) & $1.41(0.85)$ & $1.37(0.70)$ & 0.77 \\
\hline Bilirubin (mg/dl), mean (SD) & $1.79(2.30)$ & $2.02(2.3)$ & 0.58 \\
\hline Aspartate lysine aminotransferase (UI/l), mean (SD) & 359 (843) & $262(573)$ & 0.43 \\
\hline Lactate $(\mathrm{mmol} / \mathrm{l})$, mean $(\mathrm{SD})$ & $2.13(1.92)$ & $2.60(2.49)$ & 0.24 \\
\hline Left ventricular ejection fraction (\%), mean (SD) & $24(14)$ & $23(12)$ & 0.49 \\
\hline Left ventricular end-diastolic diameter (mm), & 57 (11) & 65 & 0.001 \\
\hline Cardiac index (ml/min/m2), mean (SD) & $2.17(0.77)$ & $1.94(0.7)$ & 0.20 \\
\hline Central venous pressure (mmHg), mean (SD) & $14(7)$ & $14(5)$ & 0.63 \\
\hline Capillary wedge pressure (mmHg), mean (SD) & $22(8)$ & $24(3)$ & 0.20 \\
\hline Mean pulmonary artery pressure (mmHg), mean (SD) & $28(10)$ & $30(12)$ & 0.089 \\
\hline
\end{tabular}

IABP: intra-aortic balloon pump; INR: international normalized ratio; INTERMACS: Interagency Registry for Mechanically Assisted Circulatory Support; VA-ECMO: venoarterial extracorporeal membrane oxygenation. 


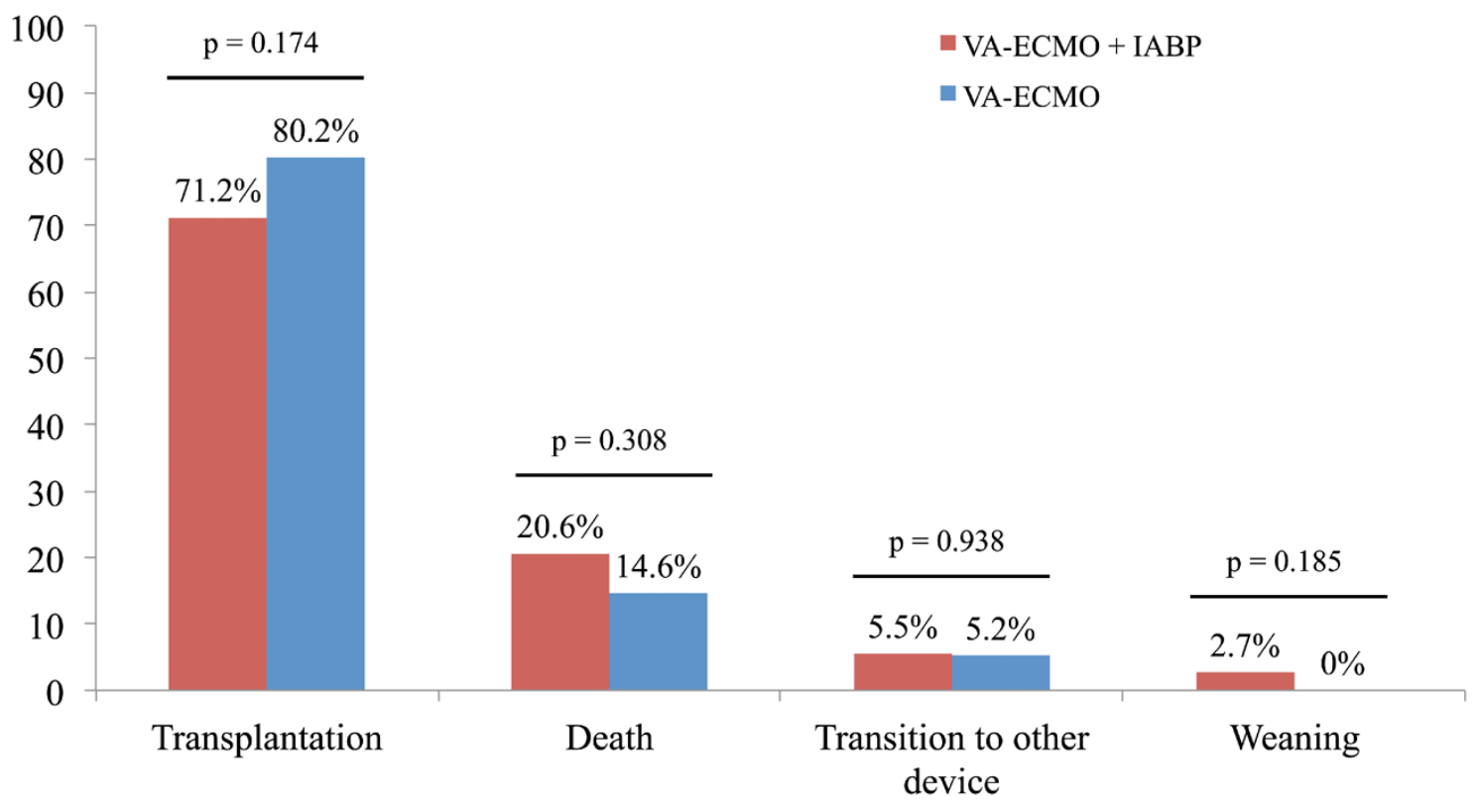

Fig. 1. Clinical outcomes of VA-ECMO support. P-value for comparison was obtained by means of the $\chi 2$ test. IABP: intra-aortic balloon pump; VA-ECMO: venoarterial extracorporeal membrane oxygenation.

Table 2: Cumulative incidence of adverse clinical events during support

\begin{tabular}{|c|c|c|c|}
\hline & VA-ECMO + IABP $(n=73)$ & VA-ECMO $(n=96)$ & P-value \\
\hline Infection, n (\%) & $23(31.5)$ & $29(30.2)$ & 0.86 \\
\hline Bleeding event, $\mathrm{n}(\%)$ & $33(45.2)$ & $24(25)$ & 0.006 \\
\hline Related to vascular access site, n (\%) & $19(26)$ & $11(11.5)$ & 0.014 \\
\hline Not related to vascular access site, n (\%) & $21(28.8)$ & $14(14.6)$ & 0.024 \\
\hline Thoracic & $11(15.1)$ & $10(10.4)$ & 0.35 \\
\hline Intracranial & $3(4.1)$ & 0 & 0.045 \\
\hline Gastrointestinal & $2(2.7)$ & 0 & 0.10 \\
\hline Urinary tract & $1(1.4)$ & 0 & 0.25 \\
\hline Skin, mucosa and soft tissue & 7 (9.6) & $3(3.2)$ & 0.078 \\
\hline Other or non-specified & $1(1.4)$ & $2(2.1)$ & 0.73 \\
\hline Stroke, n (\%) & $5(6.8)$ & $2(2.1)$ & 0.12 \\
\hline Device dysfunction, n (\%) & $5(6.8)$ & $6(6.2)$ & 0.88 \\
\hline Any major clinical adverse eventa, n (\%) & $45(61.6)$ & $46(47.9)$ & 0.076 \\
\hline Left ventricular dilation syndrome, n (\%) & $11(15.1)$ & $14(14.6)$ & 0.93 \\
\hline Venous thromboembolism, n (\%) & $2(2.7)$ & $4(4.2)$ & 0.62 \\
\hline Non-CNS arterial thromboembolism, n (\%) & $4(5.5)$ & $6(6.3)$ & 0.83 \\
\hline Renal failure requiring dialysis, n (\%) & $13(17.8)$ & $15(15.6)$ & 0.71 \\
\hline Haemolysis, n (\%) & $3(4.1)$ & $6(6.2)$ & 0.54 \\
\hline Vascular access site complication, n (\%) & $20(27.4)$ & $17(17.7)$ & 0.13 \\
\hline Pleural effusion or pneumothorax, n (\%) & $5(6.8)$ & $6(6.2)$ & 0.88 \\
\hline Pericardial effusion, n (\%) & $6(8.2)$ & $3(3.1)$ & 0.14 \\
\hline
\end{tabular}

${ }^{\mathrm{a}}$ Bleeding, stroke, infection and device dysfunction were considered major clinical adverse events in this study.

CNS: central nervous system; IABP: intra-aortic balloon pump; VA-ECMO: venoarterial extracorporeal membrane oxygenation.

\section{Adverse clinical events during venoarterial extracorporeal membrane oxygenation support}

Table 2 shows the cumulative incidence of adverse clinical events during VA-ECMO support. Bleeding events were significantly higher among patients in the IABP group compared to the control group $(45.2 \%$ vs $25 \%, \mathrm{P}=0.006)$. Significant differences were observed both in the cumulative incidence of bleeding events related to vascular access $(21.4 \%$ vs $11.5 \%, \mathrm{P}=0.024)$ and in unrelated events $(28.8 \%$ vs $14.6 \%, \mathrm{P}=0.024)$. The cumulative incidence of intracranial bleeding was $4.1 \%$ in the intervention group and $0 \%$ in the control group. By means of logistic regression, the adjusted odds ratio for bleeding events in the IABP group versus the control group was 2.18 (95\% confidence interval 1.09-4.36; $\mathrm{P}=0.028$; Supplementary Material, Table S1). 
No statistically significant differences were observed between study groups with regard to the cumulative incidence of any other adverse clinical events during VA-ECMO support. The left ventricular dilation syndrome occurred in 11 patients in the IABP group (15.1\%) and 14 patients (14.6\%) in the control group $(\mathrm{P}=0.93)$. Mechanical strategies for left ventricular unloading in patients who developed left ventricular dilation during follow-up included apical venting $(n=2)$, atrial septostomy $(n=3)$ and percutaneous left ventricular assist device implantation $(n=1)$. The remaining 19 patients who developed this complication were medically managed.

\section{In-hospital postoperative events after transplantation}

Table 3 shows the cumulative incidence of adverse clinical events during the in-hospital postoperative period after HT in 52 (71.2\%) patients in the IABP group and 77 (81.2\%) patients in the control group who were transplanted when under VA-ECMO support. At the time of transplant surgery, the IABP remained in place in 45 (86.5\%) patients in the IABP group, whereas it had been removed in 7 (13.5\%) patients. None of the 77 patients in the control group had an IABP implanted during the waiting period before transplantation.

In-hospital post-transplant mortality was $34.6 \%$ in the IABP group and $32.5 \%$ in the control group $(\mathrm{P}=0.80)$. We did not find any statistically significant differences between groups with regard to the cumulative incidence of other adverse postoperative outcomes or the length of hospital stay, as shown in Table 3.

Table 3: Cumulative incidence of in-hospital postoperative clinical outcomes following heart transplantation

\begin{tabular}{|c|c|c|c|}
\hline & VA-ECMO + IABP $(n=52)$ & VA-ECMO alone $(n=77)$ & P-value \\
\hline Primary graft dysfunction, n (\%) & $20(38.5)$ & $22(28.6)$ & 0.24 \\
\hline Right ventricular & $6(11.5)$ & $10(13)$ & \\
\hline Left ventricular or biventricular & $14(26.9)$ & $12(15.6)$ & \\
\hline Postoperative mechanical circulatory support, n (\%) & $10(19.2)$ & $11(14.3)$ & 0.46 \\
\hline Excessive surgical bleeding, $\mathrm{n}(\%)$ & $19(36.5)$ & $20(26)$ & 0.20 \\
\hline Redo cardiac surgery, n (\%) & $9(17.3)$ & $15(19.5)$ & 0.76 \\
\hline Postoperative infection, n (\%) & $25(48.1)$ & $41(53.2)$ & 0.56 \\
\hline Postoperative renal replacement therapy, n (\%) & $12(23.1)$ & $22(28.6)$ & 0.49 \\
\hline In-hospital postoperative death, $\mathrm{n}(\%)$ & $18(34.6)$ & 25 (32.5) & 0.80 \\
\hline Time on ventilator after transplant (days), mean (SD) & $11(14)$ & $12(24)$ & 0.84 \\
\hline $\begin{array}{l}\text { Time on vasoactive drug support after } \\
\text { transplant (days), mean (SD) }\end{array}$ & $8(12)$ & $8(11)$ & 0.87 \\
\hline $\begin{array}{l}\text { Time in critical care unit after transplant (days), } \\
\text { mean (SD) }\end{array}$ & $17(16)$ & $18(20)$ & 0.72 \\
\hline $\begin{array}{l}\text { Length of hospital stay after transplant (days), } \\
\text { mean (SD) }\end{array}$ & $36(35)$ & $35(44)$ & 0.82 \\
\hline
\end{tabular}

IABP: intra-aortic balloon pump; VA-ECMO: venoarterial extracorporeal membrane oxygenation.

\section{One-year survival after high-urgency listing}

Overall, 79 (46.7\%) patients died during the first year after being placed on the high-urgency waiting list, with 77 (97.4\%) patients occurring before hospital discharge. In-hospital mortality after the highurgency listing was $46.6 \%$ in the IABP group and $44.8 \%$ in the control group $(\mathrm{P}=0.82)$.

According to the Kaplan-Meier method, the estimated 1-year survival probability after the highurgency listing was $53.3 \%$ in the IABP group and $52.3 \%$ in the control group $(\log$ rank $\mathrm{P}=0.75$; Fig. 2 ).

After adjusting for potential confounders in the multivariable analysis, we observed no statistically significant effect of IABP support on the risk of death during the first year after high-urgency listing (adjusted hazard ratio 0.94, 95\% confidence interval 0.56-1.58; P=0.81; Supplementary Material, Table S2). 


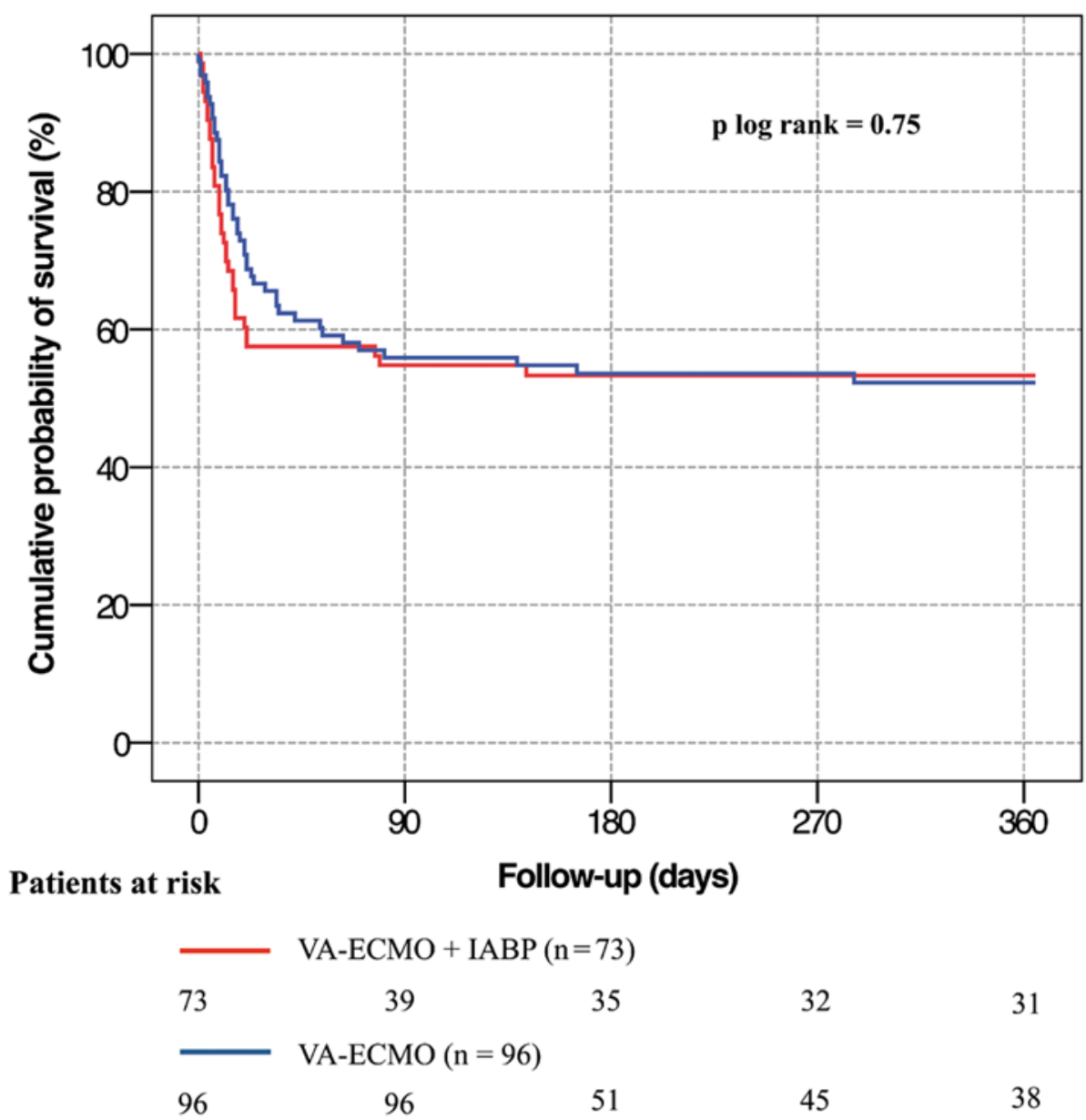

Fig. 2. Cumulative probability of 1-year survival represented by Kaplan-Meier curves. Survival curves were compared by means of the log-rank method (P-value is shown in the figure). IABP: intra-aortic balloon pump; VA-ECMO: venoarterial extracorporeal membrane oxygenation.

\section{Impact of centre volume}

The number of patients treated at each one of the participating hospitals is presented in Supplementary Material, Fig. S1. Overall, 81 (48\%) patients were treated at the 3 centres with the highest volume of procedures (institution codes 1, 11 and 16).

The ancillary use of an IABP was associated with no statistically significant impact in 1-year cumulative survival, as estimated by the Kaplan-Meier method, either in high-volume centres (IABP: $60.9 \%$ vs Control: $61.2 \%, \mathrm{P}=0.804$ ), or in low-volume centres (IABP: $50 \%$ vs Control: $38.6 \%$, $\mathrm{P}=0.430)$.

\section{Discussion}

In this article, we present a subanalysis of the Spanish multicentre ASIS-TC registry, focused on studying the safety and efficacy of the ancillary treatment with an IABP in patients listed for highurgency HT under VA-ECMO support. We did not find a significant clinical benefit of the combined therapy over VA-ECMO support alone. However, in our series, the combined VA-ECMO plus IABP therapy was found to be associated with higher bleeding risk. 
The prevention and early treatment of left ventricular dilation syndrome is an important goal in patients supported with VA-ECMO. This complication, described in up to $20-30 \%$ of cases [13], is a consequence of increased afterload and may lead to refractory pulmonary congestion and respiratory failure, including an increased risk of pulmonary bleeding and infection. To unload the left heart, several strategies have been proposed, including percutaneous atrial septostomy [14], insertion of a pigtail catheter inside the left ventricle [15], surgical apical venting [16] and simultaneous support with an IABP or left ventricular assist devices [17].

Given its accessibility and easy insertion, IABP is commonly used as an ancillary therapy to unload the left ventricle in patients under VA-ECMO support. In patients with left ventricular systolic dysfunction, IABP facilitates left ventricular ejection, leading to a moderate increase in cardiac output and a substantial reduction in left ventricular end-diastolic pressure and wedge capillary pressure [18, 19], so as to prevent pulmonary congestion. A single-centre study [5] involving 259 patients with cardiogenic shock who were supported with VA-ECMO showed a significant reduction in radiological signs of pulmonary congestion and the duration of mechanical ventilator support among patients who were treated simultaneously with an IABP and VA-ECMO compared to patients supported with VA-ECMO alone.

Despite its positive impact on haemodynamics, there is no consistent evidence of a clinical benefit of combined therapy with an IABP and VA-ECMO in terms of survival. A pooled analysis of 29 observational studies, comprising 4576 patients, showed a $10 \%$ relative reduction in the risk of in-hospital mortality among patients with cardiogenic shock who were treated with VA-ECMO and an IABP when compared with those supported solely with VA-ECMO [6]. In addition, a multicentre study based on a cohort of 1650 Japanese patients with cardiogenic shock who were supported with VA-ECMO showed a $26 \%$ relative reduction in the risk of death in patients who also were treated with an IABP compared to patients supported with VA-ECMO alone [7]. However, a pooled analysis of 16 observational studies including 1517 patients did not confirm any clinical benefit of the combined strategy, independent of the aetiology of cardiogenic shock or the temporal sequence of insertion of the 2 devices [8]. Finally, a recent systematic review of 22 observational studies with 4653 subjects detected a significant impact of simultaneous IABP support on survival only in the subgroup of patients supported with VA-ECMO due to cardiogenic shock in the setting of acute myocardial infarction, with no effect observed in the whole cohort or in patients with other causes of cardiogenic shock [9]. Transplant candidates were minimally represented in these studies. Moreover, multicentre series reporting the results of VA-ECMO as a bridge to transplantation $[10,11]$ presented no data regarding the safety and efficacy of simultaneous IABP support in this specific setting.

Similar to other studies [7, 9, 20], we were unable to find a significant clinical benefit of simultaneous IABP therapy in VA-ECMO-supported candidates for high-urgency HT, neither during the waiting period before surgery nor after transplantation. Indeed, combined support was not associated with a significant reduction in any of the studied preoperative or postoperative clinical outcomes.

It is notable that there was no significant impact of IABP support on the incidence of left ventricular dilation syndrome, which was $\sim 15 \%$ over a mean support duration of 10 days in our cohort. Patients receiving concomitant support with VA-ECMO and IABP had left ventricles that were smaller in diameter than those of the control group; however, no statistically significant differences regarding mean capillary wedge pressures were observed. This finding could be used to argue against the efficiency of IABP support to unload the left ventricle; however, this study was not specifically designed to address this question, so this finding should be interpreted with caution. Moreover, there was a higher frequency of subclavian/axillary artery cannulation in the control group, which might have contributed to a smaller increase in left ventricular afterload in these patients [21].

It should also be noted that patients treated simultaneously with IABP and VA-ECMO in our series had a more acute clinical presentation than patients treated with VA-ECMO alone, and a higher prevalence of recent myocardial infarction as the cause of shock and a higher requirement for vasoactive drugs and invasive mechanical ventilation, indicating a more severe clinical status. Indeed, the majority of patients simultaneously treated with both devices at the time of high-urgency listing were already being supported with an IABP prior to VA-ECMO implantation. Thus, it is not clear whether asymmetries in baseline clinical characteristics between study groups led to confounding bias, even though the observed statistical associations showed no substantial variation after comprehensive multivariate adjustment. 
One of the most remarkable results of our analysis was the observed independent significant association of combined therapy with an increased risk of bleeding events, regardless of whether the event was related to the vascular access site. There is no clear explanation for this observation. Previous studies have reported increased fibrinolytic activity [22] and a high incidence of thrombocytopaenia [23] in patients supported with an IABP; however, the clinical repercussions of these findings remain controversial [24].

\section{Limitations}

Our study has a few limitations. Firstly, as this study was retrospective, it is at higher risk for potential confounding, information and selection biases that are inherent to this type of investigation. Secondly, as it included only Spanish candidates for high-urgency HT, the results are not necessarily generalizable to other populations. Thirdly, given the study utilized a real-world practice-based registry, the decision and timing of IABP implantation were driven by clinical judgement and local protocols rather than by predefined criteria. Thus, it is possible that differences in routine clinical practice and experience among participating centres might have conditioned the results. Regarding this, we recognize that a mixed effects logistic regression analysis (for bleeding events) and a clustered Cox proportional hazards model (for survival) might be desirable to control potential centre-related differences in outcomes; however, the limited sample size of the study and, more importantly, the small number of patients treated at some of the participating hospitals, prevented us from attempting such statistical analyses.

Finally, although we performed a comprehensive multivariate adjustment, we cannot rule out that other confounding factors that were not measured in the current study could have affected the observed statistical associations.

\section{Conclusion}

In conclusion, our multicentre, registry-based analysis did not show a significant clinical impact of ancillary therapy with an IABP in addition to VA-ECMO support in Spanish candidates for high-urgency HT. Larger studies are required to further elucidate the best strategy for left ventricular unloading in patients supported with VA-ECMO as a bridge to transplantation.

\section{Acknowledgements}

The authors are grateful to the staff of the Organización Nacional de Trasplantes (ONT) for their collaboration in this study.

\section{Funding}

This study was funded by a research grant from the Fundación Mutua Madrileña, Madrid, Spain. A few investigators of this study are affiliated with the Centro de Investigación Biomédica en Red de Enfermedades Cardiovasculares (CIBERCV) of the Instituto de Salud Carlos III.

\section{Conflict of interest:}

Eduardo Barge-Caballero received an academic grant from Abbot Vascular (formerly Saint Jude Medical), not directly related to this investigation. There are no other relevant conflicts of interest to disclose. 


\section{References}

1 Ponikowsky P, Voors AA, Anker SD, Bueno H, Cleland JGF, Coats AJS et al. 2016 ESC guidelines for the diagnosis and treatment of acute and chronic heart failure. Rev Esp Cardiol (Engl Ed) 2016;69:1167.

2 González-Vílchez F, Almenar-Bonet L, Crespo-Leiro M, Alonso-Pulpon L, GonzalezCostello J, Sobrino-Marquez JM et al. Spanish heart transplant registry. 29th official report of the Spanish Society of Cardiology Working Group on Heart Failure. Rev Esp Cardiol (Eng Ed) 2018;71:952-60.

3 Barge-Caballero E, Almenar-Bonet L, Villa-Arranz A, Perez-Villa F, Segovia-Cubero J, Delgado-Jimenez $\mathrm{J}$ et al. Impact of short term mechanical circulatory support with extracorporeal devices on postoperative outcomes after emergency heart transplantation: data from a multi-institutional Spanish cohort. Int J Cardiol 2014;176:86-93.

4 Schiller P, Vikholm P, Hellgren L. Experimental venoarterial extracorporeal membrane oxygenation induces left ventricular dysfunction. ASAIO J 2016;62:518-24.

5 Bréchot N, Demondion P, Santi F, Lebreton G, Pham T, Dalakidis A et al. Intra-aortic balloon pump protects against hydrostatic pulmonary oedema during peripheral venoarterial-extracorporeal membrane oxygenation. Eur Heart J Acute Cardiovasc Care 2018;7:62-9.

6 Li Y, Yan S, S Liu M G, Lou S, Liu G et al. Effect of an intra-aortic balloon pump with venoarterial extracorporeal membrane oxygenation on mortality of patients with cardiogenic shock: a systematic review and meta-analysis. Eur J Cardiothorac Surg 2018; doi:10.1093/ejcts/ezy304

7 Aso S, Matsui $\mathrm{H}$, Fushimi K, Yasunaga $\mathrm{H}$ et al. The effect of intraaortic balloon pumping under venoarterial extracorporeal membrane oxygenation on mortality of cardiogenic patients: an analysis using a nationwide inpatient database. Crit Care Med 2016;44:1974-9.

8 Cheng R, Hachamovitch R, Makkar RRD, Moriguchi JD, Arabia FA et al. Lack of survival benefit found with use of intraaortic balloon pump in extracorporeal membrane oxygenation: a pooled experience of 1517 patients. J Invasive Cardiol 2015;27:453-8.

9 Vallabbaiosyula S, Horo Jc O, Antharam P. Concomitant intra-aortic balloon pump use in cardiogenic shock requiring veno-arterial extracorporeal membrane oxygenation. Circ Cardiovasc Interv 2018;11:e006930.

10 Jasseron C, Lebreton G, Cantrelle C, Legeai C, Leprince P, Flecher E et al. Impact of heart transplantation on survival in patients on venoarterial extracorporeal membrane oxygenation at listing in France. Transplantation 2016;100:1979-87.

11 Fukuhara S, Takeda K, Kurlansky PA, Naka Y, Takayama H et al. Extracorporeal membrane oxygenation as a direct bridge to heart transplantation in adults. J Thorac Cardiovasc Surg 2018;155:1607-18.e6.

12 Barge-Caballero E, Almenar-Bonet L, González-Vilchez F, Lambert-Rodriguez JL, Gonzalez-Costello J, Segovia-Cubero J et al. Clinical outcomes of temporary mechanical circulatory support as a direct bridge to heart transplantation: a nationwide Spanish registry. Eur J Heart Fail 2018;20:178-86.

13 Demondion P, Fournel L, Golmard J-L, Niculescu M, Pavie A, Leprince P. Predictors of 30-day mortality and outcome in cases of myocardial infarction with cardiogenic shock treated by extracorporeal life support. Eur J Cardiothorac Surg 2014;45:47-54.

14 Bignon M, Roule V, Dahdouh Z, Sabatier R, Lognoné T, Malcor G et al. Percutaneous balloon atrioseptostomy for left heart discharge in extracorporeal life support patients with persistent pulmonary edema. J Interv Cardiol 2012;25:62-7.

15 Barbone A, Malvindi PG, Ferrara P, Tarelli G. Left ventricle unloading by percutaneous pigtail during extracorporeal membrane oxygenation. Interact CardioVasc Thorac Surg 2011;13:293-5.

16 Fumagalli R, Bombino M, Borelli M, Rossi F, Colombo V, Osculati G et al. Percutaneous bridge to heart transplantation by venoarterial ECMO and transaortic left ventricular venting. Int J Artif Organs 2004;27:410-13.

17 Cheng A, Swartz MF, Massey HT. Impella to unload the left ventricle during peripheral extracorporeal membrane oxygenation. ASAIO J 2013;59:533-6.

18 Sauren LDC, Reesink KD, Selder JL, Beghi C, van der Veen FH, Maessen JG et al. The acute effect of intra-aortic balloon counterpulsation during extracorporeal life support: an experimental study. Artif Organs 2007;31:31-8.

19 Petroni T, Harrois A, Amour J, Lebreton G, Brechot M, Tanaka S et al. Intra-aortic balloon pump effects on macrocirculation and microcirculation in cardiogenic shock patients supported by venoarterial extracorporeal membrane oxygenation. Crit Care Med 2014;42:2075-82.

20 Lin LY, Liao CW, Wang CH, Chi NH, Yu HY, Chou NK et al. Effects of additional intraaortic balloon counterpulsation therapy to cardiogenic shock in patients supported by extracorporeal membranous oxygenation. Sci Rep 2016;6:23838. 
21 Rao P, Khalpey Z, Smith R, Burkhoff D, Kociol RD. Venoarterial extracorporeal membrane oxygenation for cardiogenic shock and cardiac arrest. Circ Heart Fail 2018;11:e004905.

22 Ficek SJ, Stammers A, Deligonul USS, Alonso A, Galbraith T et al. Hemostatic assessment of patients undergoing intraaortic balloon pump therapy. J Extra Corpor Technol 1997;29:78-82.

23 Bream-Rouwenhorst HR, Hobbs RA, Horwitz PA. Thrombocytopaenia in patients treated with heparin, combination antiplatelet therapy, and intra-aortic balloon pump counterpulsation. J Interv Cardiol 2008;21:350-6.

24 Roy SK, Howard EW, Panza JA, Cooper HA. Clinical implications of thrombocytopaenia among patients undergoing intra-aortic balloon pump counterpulsation in the coronary care unit. Clin Cardiol 2010;33:30-5. 